\title{
448 khz Capacitive Resistive Monopolar Radiofrequency in Patients with Rotator Cuff Tendinopathy. A Pilot Study
}

\section{Dimitrios Stasinopoulos $^{1 *}$, Antonis Constantinou ${ }^{2}$ and Dimitrios Lamnisos $^{3}$}

${ }^{1}$ Assistant Professor, Physiotherapy, Department of Physiotherapy, Faculty of Health and Caring Sciences, University of West Attica, Member of Laboratory of Neuromuscular and Cardiovascular Study of Motion (LANECASM)

${ }^{2}$ Physical Therapist/Clinical Trainer, Cyprus Musculoskeletal and Sports Trauma Research Centre (CYMUSTREC) Department of Health Sciences, Physiotherapy Program, School of Sciences, European University of Cyprus, Nicosia, Cyprus ${ }^{3}$ Chairperson / Assistant Professor Biostatistics, Co-Ordinator of Msc in Public Health, Department of Health Sciences, Cyprus Musculoskeletal and Sports Trauma Research Centre (CYMUSTREC) Physiotherapy Program, School of Sciences, European University of Cyprus, Nicosia, Cyprus

*Corresponding Author: Dimitrios Stasinopoulos, Assistant Professor, Physiotherapy, Department of Physiotherapy, Faculty of Health and Caring Sciences, University of West Attica, Member of Laboratory of Neuromuscular and Cardiovascular Study of Motion (LANECASM).
Received: February 29, 2020

Published: March 122020

(C) All rights are reserved by Dimitrios

Stasinopoulos., et al.

\begin{abstract}
The aim of the present pilot study was to find out the effect of different modes of $448 \mathrm{kHz}$ Capacitive Resistive Monopolar Radiofrequency in chronic rotator cuff tendinopathy. The first group received sub thermal output power (no heating effects) in continuous wave; the second group received sub thermal output power (no heating effects) in continuous modulation and the third group received thermal (thermia or hyperthermia) in continuous wave. An exercise programme was followed by all patients. Pain, function and strength were measured. Pain was decreased, function and strength were increased at the end of the treatment and at 3- and 6-month follow-ups for all modes of $448 \mathrm{kHz}$ Capacitive Resistive Monopolar Radiofrequency. The thermal (thermia or hyperthermia) in continuous wave produced the largest effect at the end of treatment and at the follow ups. Further well-designed randomized controlled clinical trials are required to determine the effectiveness $448 \mathrm{kHz}$ Capacitive Resistive Monopolar Radiofrequency in the management of chronic rotator cuff tendinopathy.
\end{abstract}

Keywords: 448 Khz; Capacitive Resistive; Otator Cuff Tendinopathy

\section{Introduction}

Tendinopathies are not only common among sports players, professional and recreational, but also among people those in jobs that involve manual labour. Tendinopathies may affect a variety of tendons including rotator cuff (mainly supraspinatus), extensor carpi radialis brevis (ECRB, commonly referred to as tennis elbow and /or lateral epicondylitis (LE)), patellar and achilles.

Rotator cuff tendinopathy (RCT) is the most common tendinopathy in the shoulder area and one of the two most common 
tendinopathies of the upper limb. Pain and decreased function are the main symptoms of RCT. Diagnosis is simple. The symptoms are reproduced by [1] overhead activities; [2] palpation on the site of pain; [3] clinical tests such as Hawkins and Neer.

The management of chronic RCT is usually conservative. Chronic RCT is failed healing tendon response or degenerative rather than inflammatory. A plethora of physiotherapy techniques such as exercise programs, soft tissue manipulation, manual techniques and electrotherapeutic modalities and have been proposed for the management of chronic RCT. Such a variety of treatment techniques suggests that the optimal treatment management is not known, and more research is required to discover the most effective treatment approach in RCT patients. More recently, physiotherapists are able to use a new modality called $448 \mathrm{kHz}$ Capacitive Resistive Monopolar Radiofrequency (CRMRF). However, the evidence of the $448 \mathrm{kHz}$ CRMRF in the management of chronic RCT, is minimal. Therefore, the objective of the present pilot study is to find out the effect of different modes of $448 \mathrm{kHz}$ CRMRF in chronic RCT.

\section{Methods}

A monocenter pilot trial was conducted in a research centre over 8 months to assess the effectiveness of $448 \mathrm{kHz}$ CRMRF in patients with chronic RCT. Three investigators were involved in the study: [1] the primary investigator who administered the treatments (DS); [2] a specialized orthopaedic who had over 15 years' experience and who evaluated the patients to confirm the RCT diagnosis; and [3] a PhD physiotherapy student (AC), who performed all baseline and follow-up assessments, and gained informed consent. AC who was blind to the patients' therapy group conducted all assessments. Each patient to ascertain baseline demographic and clinical characteristics, including sex, age patient, name, duration of symptoms, affected arm and dominant arm, occupation and previous treatment were interviewed by AC.

Patients over 18 years old with shoulder pain were examined and evaluated in the Cyprus Musculoskeletal and Sports Trauma Research Centre located in Nicosia between April 2018 and July 2018. All subjects lived in Nicosia, Cyprus, were native speakers of Greek, and were either referred by their physician or physiotherapist or self-referred.

Subjects were included in the study if, at the time of presentation, they had been evaluated as having clinically diagnosed RCT for at least four weeks. Subjects were included in the study if they reported positive hawkins, neer and epty can position test and pain on palpation (upper aspect of the head of the humerus) [1]. Patients were excluded if they: [1] reported a history of glenohumeral dislocation, or other traumatic injury to the shoulder; [2] had a history of rotator cuff surgery; [3] had shoulder symptoms reproduced by a cervical assessment or [4] reported only periscapular or cervical pain during arm elevation;. In addition, to detect calcifications and whether there were signs of arthrosis in the acromio-clavicular joint an x-ray was performed. Subjects with signs of arthrosis in the acromioclavicular joint and/or calcifications in the rotator cuff were also not included in this pilot trial.

A written explanation of the trial were received by all subjects before entry into the study and then gave signed consent to participate. They were allocated to three groups; sub thermal output power (no heating effects) in continuous wave; sub thermal output power (no heating effects) in continuous modulation and thermal (thermia or hyperthermia) in continuous wave by drawing lots.

All subjects were instructed to use their arm during the period of the study but to avoid activities that irritated the shoulder such as sleep on the affected shoulder, quick movements of the shoulder and full elevation of the shoulder. They were also told to refrain from taking pain killer drugs throughout the course of trial. Subject compliance with the above mentioned request was monitored using a treatment diary.

A qualified physiotherapist (DS) with about 20 years' experience in the management of tendinopathies administered all treatments at the centre. interaction (verbal and non-verbal) and communication between the therapist and subject were kept to a minimum and behaviors sometimes used by clinicians to facilitate positive treatment outcomes were purposefully avoided.

Patients received $448 \mathrm{kHz}$ CRMRF intervention. The CRMRF at $448 \mathrm{kHz}$ was delivered using 'INDIBA Activ 902', a new factory calibrated device with a peak power of $200 \mathrm{~W}$ and 450VA, which delivered continuous-wave or continuous modulation RF energy in two modes: Capacitive (CAP) and Resistive (RES), using metallic electrodes via a coupling medium. The CAP mode was delivered in thermal dose (according to patient feedback on his perception of moderate heating) in muscles around the shoulder. CAP mode was delivered 5 minutes for each muscle. The RES mode was delivered in sub thermal output power (no heating effects) in continuous wave for first group; in sub thermal output power (no heating ef-

Citation: Dimitrios Stasinopoulos., et al. "448 khz Capacitive Resistive Monopolar Radiofrequency in Patients with Rotator Cuff Tendinopathy. A Pilot Study”. Acta Scientific Orthopaedics 3.4 (2020): 16-20. 
fects) in continuous modulation for the second group and thermal (thermia or hyperthermia) in continuous wave for third group. The RES mode was delivered for 10 minutes. Finally, CAP mode in nonthermal dose was delivered in the symptomatic area for 5 minutes. The return electrode was placed in the scapular area. Treatment was delivered once per day for five consecutive days providing twenty sessions in total.

An exercise programme was followed by subjects in all (three) groups followed daily (apart from weekends) for 4 weeks [2]. The strengthening exercises were included (i) shoulder lateral rotation and medial with the elbow in 900 and 00 of abduction; (ii) shoulder abduction to 900 with flexed elbow: (iii) scaption and (iv) diagonal pattern from flexion to extension. Upper trapezius, rhomboids, serratus anterior and levator scapulae were also strengthened [2,4]. Each exercise were carried out twice at each treatment with 12 repetitions in each set and 1 min rest interval between each set. Subjects were informed to continue with the exercise even if they complained of mild pain. However, subjects were informed to stop the exercise if the pain became disabling. When subjects were able to carry out the strengthening exercises without experiencing any discomfort or pain, the load were increased using therabands or free weights. Static stretching exercises including (i) external rotators of the shoulder and (ii) inferior and posterior capsule stretch according to prentice (1999) [3]. Static stretching exercises were repeated three times at each treatment session, after the strengthening exercises with a 30-s rest interval between each repetition. 30 - 45 s was held each stretching for each time and then released. The exercise programme treatment was individualized one the basis of the subject's description of discomfort experienced during the procedure.

Pain, Function, strength and dropout rate were measured in this study. Each patient was evaluated at the baseline (week 0), at the end of treatment (week 4) three months (week 12) and six months (week 24) after the end of treatment in order to see the short, intermediate and long-term effects of the treatments. Pain and function were measured using the Greek version of Shoulder Pain and Disability Index (SPADI) which is a reliable and valid measure when administered to patients aged over 18 years old with shoulder pain for at least 4 weeks [4]. Strength was measured using the hand held dynamometer according to Savva., et al. (2018) [5] which is a valid and reliable outcome measures for patients with RCT. As an indicator of treatment outcome was also used the dropout rate. Dropouts were categorised as follows: (a) did not return for follow up; (b) request for an alternative treatment; and (c) withdrawal without reason.

The change from the beginning of the treatment will be calculated for each follow-up. Differences in this change pain and function on the SPADI and change in pain-free grip strength was calculated between the groups and was determined using Kruskal wallis test. Mann - whitney test was used to measure the improvement in all groups when compared with the pretreatment baseline. Bonferroni post-hoc comparisons were conducted when the results from the Kruskal wallis test were significant to determine how the three groups differed. The level for statistical significance were adopted as a $5 \%$ level of probability. The statistical analysis were carried out using the SPSS 21.00 statistical software.

\section{Results}

19 patients eligible for inclusion visited the research centre within the treatment session. Two were unwilling to took part in the trial and 2 did not meet the above mentioned inclusion criteria. The other 15 patients were allocated into one of the three possible groups: [1] subthermal output power (no heating effects) in continuous wave ( $\mathrm{n}=4 ; 2$ male, 2 female; mean age $=45.6$ years + -SD $=4.5$ years), [2] sub thermal output power (no heating effects) in continuous modulation $(\mathrm{n}=5 ; 2$ male, 3 female; mean age $=46.7$ years + _SD $=4.4$ years) and [3] thermal (thermia or hyperthermia) in continuous wave $(n=6 ; 3$ male, 3 female; mean age $=45.5$ years + +_SD $=3.2$ years).

The mean age of patients was approximately 45 years and the duration of RCT was approximately six months. $90 \%$ of subjects had RCT in the dominant arm in. There were no significant differences in mean age $(\mathrm{P}<0.0005$, Kruskal wallis test) or the mean duration of complaints ( $\mathrm{P}<0.0005$, Kruskal wallis test) between the groups. All subjects had tried drug therapy. All subjects were manual workers.

Baseline SPADI was 85 (95\% confidence interval $(\mathrm{CI})=77$ - 93) for the whole sample $(n=15)$. There were no significant differences between the groups for SPADI at baseline (P > 0.05 Kruskal wallis test). At week 4 (end of treatment) there was a decline in SPADI of approximately 70 units in three (all) groups when compared with the pretreatment baseline ( $\mathrm{P}<0.0005$ Mann - whitney test). There was a significant difference in the magnitude of reduction between the groups ( $\mathrm{P}<0.0005$ Kruskal wallis test), so post-hoc tests were 
performed. The magnitude of reduction was significantly larger for the thermal (thermia or hyperthermia) in continuous wave than sub thermal output power (no heating effects) in continuous wave (+5 units) and sub thermal output power (no heating effects) in continuous modulation ( +7 units, $\mathrm{P}<0.05$, Bonferroni). There was no significant difference between sub thermal output power (no heating effects) in continuous wave and sub thermal output power (no heating effects) in continuous modulation (+2 units, $\mathrm{P}>0.05$, Bonferroni,). Similarly at week 12 and week 24 there were comparable magnitudes of reduction with larger reduction for the thermal (thermia or hyperthermia) in continuous wave than for sub thermal output power (no heating effects) in continuous wave and for sub thermal output power (no heating effects) in continuous modulation ( $\mathrm{P}<0.05$, Bonferroni,). There was not a significant difference between sub thermal output power (no heating effects) in continuous wave and sub thermal output power (no heating effects) in continuous modulation ( $\mathrm{P}>0.05$, Bonferroni).

Baseline grip strength was $29.6 \mathrm{lb}$ (95\% confidence interval (CI) $=26.2-31.3)$ for the whole sample $(n=15)$. No significant differences were found between the groups at baseline grip strength $(\mathrm{P}>$ 0.05 Kruskal wallis test). At week 4 there was a rise in grip strength of approximately 30 units in all groups when compared with the pretreatment baseline $(\mathrm{P}<0.0005$ Mann - whitney test). There was a significant difference in the magnitude of improvement between the groups ( $\mathrm{P}<0.0005$ Kruskal wallis test), so post-hoc tests were performed. The magnitude of improvement was significantly larger the thermal (thermia or hyperthermia) in continuous wave than sub thermal output power (no heating effects) in continuous wave (+ 7 grip strength units) and sub thermal output power (no heating effects) in continuous modulation (+ 8.5 grip strength units, $\mathrm{P}<$ 0.05 , Bonferroni). There was no significant difference between sub thermal output power (no heating effects) in continuous wave and sub thermal output power (no heating effects) in continuous modulation (+ 2.5 grip strength units, $\mathrm{P}>0.05$, Bonferroni). Similarly at week 12 and week 24 there were comparable magnitudes of improvement with larger improvements for the thermal (thermia or hyperthermia) in continuous wave than for sub thermal output power (no heating effects) in continuous wave and for sub thermal output power (no heating effects) in continuous modulation $(\mathrm{P}<$ 0.05 , Bonferroni). There was not a significant difference between sub thermal output power (no heating effects) in continuous wave and sub thermal output power (no heating effects) in continuous modulation ( $\mathrm{P}>0.05$, Bonferroni).
All patients successfully completed the study, as no drop-outs were reported.

\section{Discussion}

The results obtained from this pilot trial are novel, as to date there have been no data comparing the effectiveness of different modes of $448 \mathrm{kHz}$ CRMRF such as sub thermal output power (no heating effects) in continuous wave, sub thermal output power (no heating effects) in continuous modulation and thermal (thermia or hyperthermia) in continuous wave in chronic RCT. The thermal (thermia or hyperthermia) in continuous wave produced the largest effect at the end of treatment and at the follow ups.

Two types of exercise programs exist: exercise programs carried out in a clinical setting and home exercise programs. A home exercise program is commonly advocated for subjects with tendon injuries such as RCT because it can be carried out any time during the day without requiring supervision by a clinician. Our clinical experience, however, has shown that patients fail to comply with the regimen of home exercise programs [6] This problem can be solved by exercise programs performed in a clinical setting under the supervision of a physiotherapist. For the purposes of this report, "supervised exercise program" will refer to such programs. Therefore, such a supervised exercise program was used in the present trial.

Although a supervised exercise program is an effective treatment approach, a supplement to the exercise program should be found to reduce the treatment period. One such modality is 448 $\mathrm{kHz}$ CRMRF which is a relatively new treatment approach, but it is reported to be used by clinicians worldwide. Many clinicians think that Shortwave Diathermy (SWT) and $448 \mathrm{kHz}$ CRMRF is the same. However, the $448 \mathrm{kHz}$ CRMRF differs from SWT mainly in two ways - firstly the operating frequency (SWT commonly operates at 27.12 $\mathrm{MHz}$ ) and secondly, unlike SWT it is applied using a coupling medium since CRMRF cannot be delivered through air [7]. Hence, one hypothesized advantage of $448 \mathrm{kHz}$ CRMRF over SWT is that scattering of the RF waves is potentially considerably lower [7].

Since pain relief and improvements in function and strength were noted in the present study on a long term, it is proposed that the $448 \mathrm{kHz}$ CRMRF energy may potentially have promoted an important effect in the management of soft tissues [7]. However, to understand the potential changes to the tissues in response to 448 $\mathrm{kHz}$ CRMRF treatment, future studies should consider employing outcome assessments that are capable of monitoring the changes in deeper tissues.

Citation: Dimitrios Stasinopoulos., et al. "448 khz Capacitive Resistive Monopolar Radiofrequency in Patients with Rotator Cuff Tendinopathy. A Pilot Study". Acta Scientific Orthopaedics 3.4 (2020): 16-20. 
The present pilot trial was the first trial to examine the effectiveness of $448 \mathrm{kHz}$ CRMRF on chronic RCT. One previous study assessed the effectiveness of this treatment on chronic knee osteoarthritis [7]. However, RCT and knee osteoarthritis are two different conditions and the results are not comparable. The two previously reported trials found that a course of $448 \mathrm{kHz}$ CRMRF may improve patients' symptoms. The findings of these two trials encourage the design of future well-designed RCTs that might produce strong evidence for the effectiveness of $448 \mathrm{kHz}$ CRMRF on sports/musculoskeletal injuries.

A course of $448 \mathrm{kHz}$ CRMRF treatment was applied in the present pilot study based on manufacturers' claims. It is a dose-response modality and the optimal treatment dose has obviously not yet been discovered. Future studies are needed to standardize 448 $\mathrm{kHz}$ CRMRF parameters in the management of RCT (acute, chronic and calcific).

However, this pilot trialdoes have some shortcomings. First, the sample was small. Second, no treatment group or no sham (placebo) was included in the present pilot study. The no treatment / sham (placebo) group is important when the absolute effectiveness of a treatment is assessed. However, the absolute effectiveness of method based interventions is difficult to find out because a trustworthy and good no treatment / sham (placebo) control for many physiotherapy methods appears to be impossible or difficult to devise, due in part to difficulties in defining the active element of these methods. Absolute effectiveness also does not provide the clinicians with information as to which is the most appropriate method for the treatment of a condition, in this case RCT. Third, other activities treatments patients might be getting when not in the clinic were not monitored. Subjects' diaries suggested that subjects were compliant to the trial orders, although subjects may have given incorrect answers to please the researchers. For example, it was possible that subjects followed the treatment but received pain killer drugs at the same time, and the improvement of symptoms may be due to those drugs. Therefore, ways should be discovered to measure how other treatments such as pain killer drugs contribute to the improvement of symptoms. Finally, the blinding of subjects and clinician would be problematic in that trial, if not impossible, because subjects know if they are receiving the exercise programme treatment and clinicians need to be aware of the treatment to administer it cotrrectly. More research is required to determine the possible mechanism of action of this modality, and the cost-effectiveness of such technique, because reduced cost is an important factor for the recommendation of any given technique.

\section{Conclusion}

This pilot trial showed that the thermal (thermia or hyperthermia) mode of $448 \mathrm{kHz}$ CRMRF in continuous wave, had reduced the pain and improved function and strength in patients with chronic RCT at the end of the treatment and at the follow-ups. However, further well-designed randomized controlled clinical trials are required to determine the effectiveness and the mechanism of action of $448 \mathrm{kHz}$ CRMRF in chronic RCT. In addition, a cost effectiveness analysis should be incorporated into the analysis of the effectiveness of $448 \mathrm{kHz}$ CRMRF in a future study, because reduced costs are important issues for the recommendation of a technique.

\section{Bibliography}

1. Factor D and Dale B. "Current concepts of rotator Cuff tendinopathy". The International Journal of Sports Physical Therapy 9.2 (2014): 274-288.

2. Stasinopoulos D., et al. "A pilot trial to study the effectiveness of an exercise programme in the treatment of rotator cuff tendinopathy". JBE 10.2 (2014): 69-77.

3. Prentice W. "Rehabilitation techniques in sports medicine". 3rd ed. Boston: WCB/mcgraw-hill, (1999).

4. Spanou A., et al. "Reliability and validity of the Greek shoulder pain and disability index in patients with shoulder pain". Disability and Rehabilitation 17 (2019): 1-6.

5. Savva C., et al. "Test-Retest Reliability of Handgrip Strength as an Outcome Measure in Patients With Symptoms of Shoulder Impingement Syndrome". Journal of Manipulative and Physiological Therapeutics 41.3 (2018): 252-257.

6. Stasinopoulos D and Stasinopoulos I. "Comparison of effects of Cyriax physiotherapy, a supervised exercise programme and polarized polychromatic non-coherent light (Bioptron light) for the treatment of lateral epicondylitis". Clinical Rehabilitation 20 (2006): 12-23.

7. Kumaran B and Watson T. "Treatment using 448kHz capacitive resistive monopolar radiofrequency improves pain and function in patients with osteoarthritis of the knee joint: a randomised controlled trial”. Physiotherapy 105.1 (2019): 98-107.

\section{Assets from publication with us}

- Prompt Acknowledgement after receiving the article

- Thorough Double blinded peer review

- Rapid Publication

- Issue of Publication Certificate

- High visibility of your Published work

Website: www.actascientific.com/

Submit Article: www.actascientific.com/submission.php

Email us: editor@actascientific.com

Contact us: +919182824667 